\title{
KNOWLEDGE DISCOVERY AND FRAMEWORK FOR PURCHASE BEHAVIOR ANALYSIS IN MOBILE GAMING APPLICATIONS
}

\author{
Martins Jansevskis and Kaspars Osis \\ Faculty of Engineering, Vidzeme University of Applied Sciences, Valmiera, Latvia
}

\begin{abstract}
The gaming industry is a fast expanding industry with a large global market and is projected to hit $\$ 300$ billion by 2025 . The total number of players in 2019 was projected to hit 2.4 billion (Taylor, 2019). Knowledge discovery is achieved by collecting and analyzing data from within the gaming applications. The data collected is used to understand players, gain insights and to improve products for the gaming community based on feedback and user interaction. And as a result, obtained knowledge contributes to monetization in a way that is especially interesting in the e-sports and streaming space. Machine learning is one of the technologies that can assist in knowledge discovery as it provides potential to obtain insights into previously overlooked data. This paper provides insight in a work of how machine learning algorithms are applied to gain behavior understanding whithin mobile gaming applications in a way compliant with European General Data Protection Regulation (GDPR). The paper is a part of a larger research work and contributes to the domain of knowledge discovery within in-app purchase behavior data and serves as a step towards further research in this area.
\end{abstract}

\section{KEYWORDS}

Knowledge Discovery, Data Analysis, GDPR, Mobile Gaming Applications

\section{INTRODUCTION}

In mobile games usually exists an imbalance between players that spend money and those who do not. The vast majority of players are the ones that do not. One of the key challenges in mobile games is to convert players from non-spending to spending, and by doing so ensuring that the lifetime value (LTV) of players is higher than the User Acquisition Cost (UAC) (Sifa et al., 2015). To increase LTV and lower UAC, knowledge discovery by collecting and analyzing data from within the game in a manner that would be compliant with the European General Data Protection Regulation (GDPR) has to be implemented in a way that would satisfy players. The goal of the knowledge discovery process for this research is to determine if existing players or devices would ever become a paying customers and what behavioral incentives increase LTV.

In accordance to address the defined challenges, authors defined three main tasks - determine and define the potential data set that can be collected without violating GDPR, develop mobile packages to integrate into existing games, collect data to carry out analysis and apply machine learning (ML) algorithms. In the process authors were adopting methods that rely on publicly available ML algorithms, to keep results relevant for the gaming industry. The proposed framework is based on prior research in the field of knowledge discovery using machine learning (Jansevskis and Osis, 2018) and applies the previously introduced approach for potential business use. The results presented are the preparatory steps in providing a potential solution for determining if a player will contribute to a company's revenue streams. 


\section{DEVELOPMENT PROCESS}

As initially defined, knowledge discovery in this research resolves around predicting if existing players will become paying customers. To carry out this research with long term goals authors have chosen to develop a framework that would accumulate data, provide mobile packages and integrate in existing mobile gaming applications as well as develop machine learning solutions to further carry out the learning process.

\subsection{Dataset}

The research included determining potential datasets that could be used for developing the ML algorithms. Authors based their assumptions on similar researches (Sifa et al., 2015) (Han and Windsor, 2013) (Pantano and Priporas, 2016) (Xudong et al., 2016) and potentially available data from within the game. Authors chose to collect data of player's behavior in the following categories: sessions, product views, and purchases. The session is being registered when a player launches the game with the included author's developed package. The product view is being registered when a player's mobile device requests product data from mobile packages. The purchase is registered when a player spends money on in-game merchandise and creates an order. To make this research repeatable in the long term, authors chose to connect mobile packages with an online store and provide different products for purchasing. The items added to the online store and on sale are t-shirts, socks, gift-cards and 3D figurines with custom made designs for a specific game. Table. 1 lists dataset authors were including in mobile packages.

Table 1. Defined metrics dataset for behavior analysis

\begin{tabular}{lll}
\hline Category & Type & Example \\
\hline Device data & Device & iPhone10.6 \\
& OS version & 9,1 \\
& Region & en_US \\
Apame data & Apple/Google Pay status & Active \\
& Category & Puzzle \\
& Publisher & Blizzard \\
& Total number of players & 1224 \\
\hline Design & Date of install & $2020-02-20$ \\
& Date of update & $2020-02-20$ \\
& Trigger & t-shirt \\
\hline User & Game time & 455 \\
& Last session & $2020-02-20$ \\
& Session time & 707 \\
& Start of session & $2020-02-20$ \\
& End of session & $20: 20: 06.56554+02$ \\
& Time until first product view & 45 \\
\hline Encounters & Dismissal clicks & 3 \\
& Number of dismissals & 45 \\
& Dismissal ratio & 1 \\
\hline Purchase behavior & Spending amount & 6,52 \\
& Number of purchases & 1 \\
& Currency & USD \\
\hline Customer & City & Atlanta \\
& State & Georgia \\
& Country & US \\
& Product & t-shirt \\
\hline
\end{tabular}

The defined dataset for mobile packages had to be GDPR (General Data Protection Regulation (GDPR), 2020) compliant and must not include personal information. Since real purchases are being made and the order fulfillment process has to be carried out it is required to collect personal data. To accomplish GDPR compliance authors were using an online store from the Shopify platform (Shopify, 2020) and does not include data with the player's name, age, gender and detailed address in the dataset for analysis.

\subsection{Proof of Concept Algorithm Development}

The development process of potential purchase behavior algorithms included data pre-processing, outliers` removal, category variables encoding and variable normalization. The main task at this phase was removing features without enough data, removing features with constant values and removing features that can be only 
calculated after purchase has been made. Feature engineering was applied to derive new features from the historical data of player's interactions with a game. A skewed distribution towards non-paying behavior was expected as from data it was known that only a small amount of players are the paying customers (Sifa et al., 2015). To solve the problem of imbalanced datasets, it was possible for testing purposes to synthetically generate paying players following Synthetic Minority Over-sampling Technique-Nominal Continuous (SMOTE-NC) method (Chawla et al., 2002). The correlation analysis on the dataset was applied to evaluate which features were relevant to improve player engagement in the game (an increase of LTV). Feature selection techniques were used to limit the number of features by selecting only the important ones.

For evaluation the F-Score (F1-Measure) (DeepAI, 2020) the harmonic mean over the precision and recall values for predicting the paying players was used. Additionally, the geometric mean of the classification accuracy values for each of the classes (G-mean) (DeepAI, 2020) was also applied. For the baseline solution the following models have been chosen for training:

- decision trees with reduced error pruning;

- support vector machines with polynomial and radial basis function kernels;

- random forests by selecting the square root of number of possible features.

Of particular interest in the process was to determine the features that have a significant impact on predicting if player will be a paying customer.

Exists two main approaches of collaborative filtering which recommend products based on history and content-based, which is based on product descriptions and how they match with the player's profile. The advantage of the first solution is described in details with matching products to customers (Candellier et al., 2009), the main disadvantage is that the player has to have historical data.

Collaborative filtering does have a higher accuracy and is being developed based on deep learning methods (Chen, Lin, and Pan, 2017). This approach faces another challenge - the problem of scalability. New models on top of costly methods used in collaborative filtering introduced the time cost of using neural networks. The solution to this challenge with new methods of sampling was presented in research (Chen, Lin, and Pan, 2017). In the work of Weia et al. (2017) an approach of solving cold start (players without historical data) challenge has been proposed.

Based on research papers the baseline solution was developed on item-item collaborative filtering. In this phase product is a content triggered by a developed SDK. Purchasing products is a desired interaction with the given player.

\subsection{Technology Framework}

In process of planning and designing the framework for purchase behavior analysis authors carried out mobile gaming market analysis to determine potential technology stack that would cover the most common technologies used for mobile games (MarketWatch, 2020). Figure 1 shows the initial framework proposed by authors to implement player purchase behavior analysis. Proposed technology framework roots in the earlier research work done in the field of knowledge discovery and further elaborates previously proposed technological stack solutions framework (Jansevskis and Osis, 2018). The framework consists of five components that support data gathering and is designed around central data storage that is being accessed through application programming interface (API).

Data storage in the proposed framework provides functionality such as data continuity, integrity, and availability as those considerations have to be taken into account. The primary task of a cloud storage facility is to deliver data for connected mobile packages. As there are significant volumes of data produced by mobile games and player`s devices the main task was to choose solutions that can be easily extended and scaled.

Security considerations and role-based access controls (RBAC) had to be implemented between all of the components of the frameworks. To cover user privileges requirements authors propose to use OAuth 2.0 (Oauth.net, 2020) industry-standard protocols for every connected mobile software development kit (SDK) and limit access to read-only scopes for public API access. To cover data security considerations authors were using building security in the maturity model (BSIMM) (Bsimm.com, 2020). 


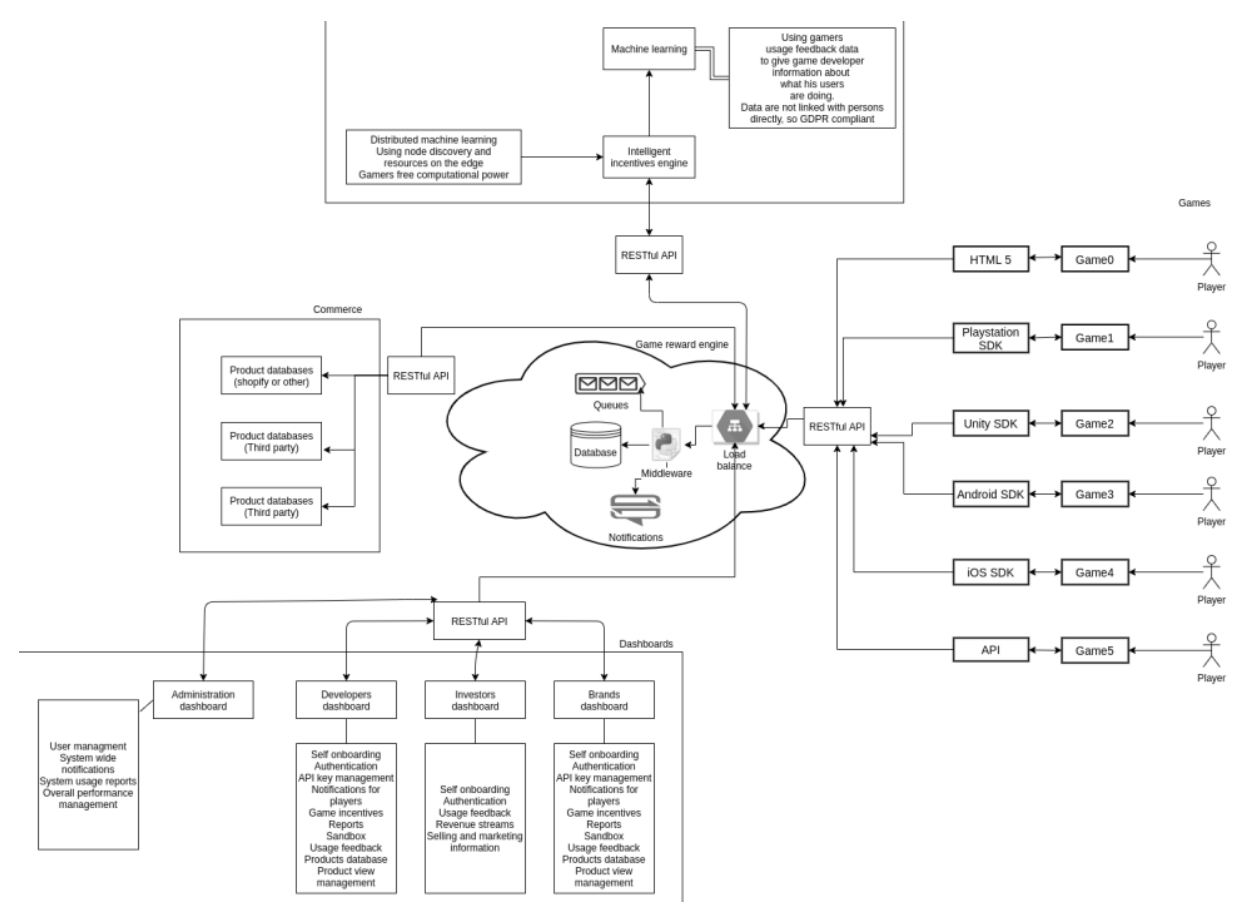

Figure 1. Proposed technology framework for purchase behavior analysis in mobile gaming applications

As data has to be collected from within the mobile gaming applications, SDKs for most common platforms have to be developed and connected to public API. In framework development process authors were including Android, iOS and Unity platforms. As in-app purchases data had to be collected authors were using Apple Pay (Apple, 2020), Google Pay (Pay.google.com, 2020) and Stripe (Stripe.com, 2020) integrations for mobile platforms.

\subsection{Algorithm Results}

To test the developed algorithm a dataset had to be prepared based on initially defined available metrics. The primary key in available data was device identifier that identifies a unique device. Based on device identifier information about purchases from stores was extracted and used as a target variable for learning task. In the preprocessing stage a subset of data was prepared using data from two-week time span and a dataset with more than 50 thousand records containing about 500 monetized devices. Learning models were tested using balancing. That was necessary because dataset contained a high imbalance as only approximately $1 \%$ of devices were monetized.

Two approaches of balancing were applied - SMOTE_NC and Random OverSampling. Following classification models were used: Dummy Classifier, AdaBoost Classifier, Logistic Regression and Random Forest Classifier. The AdaBoost Classifier model with Random OverSampling balancing method provided the most promising results with accuracy 0.854158 . For this model $85.03 \%$ of true negatives and $0.39 \%$ of true positives were returned. This indicated that approximately $50 \%$ of monetized devices were correctly recognized, while just a small percentage of non-monetized were falsely recognized.

\section{CONCLUSION}

In this paper, a framework is being described that aims to gather in-game purchase data and described the ML algorithms development process to further analyze player's behavior to determine if one is going to become a paying customer. The described framework and its related mobile SDKs have been implemented in three existing games. The ML algorithms have been applied to collected data and further research has to be carried out to determine if applied techniques and chosen algorithms hold business value. 
To advance the development of the research, it is necessary to apply framework to a larger set of mobile games and carry out further research on ML algorithms and player purchase behavior analysis. The most important and challenging task is the attempt to onboard mobile game developers and publishers to apply this framework.

\section{REFERENCES}

Apple. (2020). Apple Pay. [online] Available at: https://www.apple.com/apple-pay/ [Accessed 16 Feb. 2020].

Bsimm.com. (2020). Building Security In Maturity Model | BSIMM. [online] Available at: https://www.bsimm.com/ [Accessed 15 Feb. 2020].

Chawla, N., Bowyer, K., Hall, L. and Kegelmeyer, W. (2002). SMOTE: Synthetic Minority Over-sampling Technique. Journal of Artificial Intelligence Research, 16, pp.321-357.

Candiller, L., Jack, K., Fessant, F., i Meyer, F. (2009). State of the Art Recommender System. DOI: 10.4018/978-160566-306-7.ch001.

Chen, L., Lin, M. and Pan, Y. (2017). Find crucial factors of in-game purchase using neural networks. 2017 IEEE 8th International Conference on Awareness Science and Technology (iCAST), [online] pp.334-338. Available at: https://ieeexplore.ieee.org/document/8256473 [Accessed 20 Feb. 2020].

DeepAI. (2020). F-Score. [online] Available at: https://deepai.org/machine-learning-glossary-and-terms/f-score [Accessed 20 Feb. 2020].

General Data Protection Regulation (GDPR). (2020). General Data Protection Regulation (GDPR) - Official Legal Text. [online] Available at: https://gdpr-info.eu/ [Accessed 10 Jan. 2020].

Han, B. and Windsor, J. (2013). An investigation of the smartphone user's in-game purchase intention. International Journal of Mobile Communications, 11(6), p.617.

Jansevskis, M. and Osis, K., 2018. Machine Learning and on 5G Based Technologies Create New Opportunities to Gain Knowledge. 2018 2nd European Conference on Electrical Engineering and Computer Science (EECS).

MarketWatch. (2020). Mobile Gaming Market 2019 Global Industry Analysis, Emerging Technology, Sales Revenue, Competitive Landscape, Emerging Technologies, Gross Margin Regional Forecast To 2023. [online] Available at: https:/www.marketwatch.com/press-release/mobile-gaming-market-2019-global-industry-analysis-emergingtechnology-sales-revenue-competitive-landscape-emerging-technologies-gross-margin-regional-forecast-to-20232019-05-09 [Accessed 10 Feb. 2020].

Oauth.net. (2020). OAuth 2.0 - OAuth. [online] Available at: https://oauth.net/2/ [Accessed 5 Feb. 2020].

Pantano, E. and Priporas, C. (2016). The effect of mobile retailing on consumers' purchasing experiences: A dynamic perspective. Computers in Human Behavior, [online] 61, pp.548-555. Available at: https://www.sciencedirect.com/science/article/pii/S0747563216302448?via\%3Dihub [Accessed 20 Feb. 2020].

Pay.google.com. (2020). Google Pay (UK) - Pay in apps, on the web, and in stores. [online] Available at: https://pay.google.com/about/ [Accessed 16 Feb. 2020].

Sifa, R., Hadiji, F., Runge, J., Drachen, A., Kersting, K., Bauckhage, K (2015). Predicting Purchase Decisions in Mobile Free-to-Play Games. Proceedings, The Eleventh AAAI Conference on Artificial Intelligence and Interactive Digital Entertainment (AIIDE-15), [online] pp.79-85. Available https://pdfs.semanticscholar.org/9801/b69ac1faaffafecfea250ec65a5ac33cd8af.pdf [Accessed 20 Feb. 2020].

Shopify. (2020). Start, grow, and scale your business - Shopify. [online] Available at: https://www.shopify.com/ [Accessed 25 Feb. 2020].

Stripe.com. (2020). Stripe Payments: A complete payments platform, engineered for growth. [online] Available at: https://stripe.com/en-my/payments [Accessed 17 Feb. 2020].

Taylor, M. (2019). 'The Most Exciting Tech Niche For 2020'. [online] Bloomberg. Available at: https://www.bloomberg.com/press-releases/2019-12-30/the-most-exciting-tech-niche-for-2020 [Accessed 20 Feb. 2020].

Wei, J., He, J., Chen, K., Zhou, Y., Tang, Z. (2017). Collaborative filtering and deep learning based recommendation system for cold start items. DOI: 10.1016/j.eswa.2016.09.040

Xudong, L., Meijiao, G., Kangqing, L. and Zhiwei, Q. (2016). Analyzing the influencing factors of mobile game consumption. 2016 13th International Conference on Service Systems and Service Management (ICSSSM), pp.1-6. 